\title{
Pericardial involvement in systemic sclerosis
}

\author{
R J Byers, D A S Marshall, A J Freemont
}

\begin{abstract}
Objective-To determine the frequency and histological characteristics of pericardial involvement in systemic sclerosis. Method-Necropsy sections of pericardium from 44 patients with systemic sclerosis were studied, together with sections from 19 age/sex matched controls. Sections were stained with haematoxylin and eosin, acid toluidine blue, and elastic van Gieson. Mast cells were counted in $\mathbf{1 0}$ random high power fields and the degree of fibrosis was quantified using a Chalkley count.

Results-Chronic pericarditis was seen in $31(77.5 \%)$ of the systemic sclerosis cases, but in only one of the controls. The characteristic changes of uraemic pericarditis were not seen. The degree of fibrosis was greater in those with systemic sclerosis, though numbers of mast cells, thought to be important in fibrogenesis, were similar in both groups. Myocardial fibrosis was seen in $15(37.5 \%)$ of systemic sclerosis cases but in none of the controls. Conclusion-The incidence of pericarditis and myocardial fibrosis is much greater than in controls. The results indicate that pericarditis is a primary disease (rather than secondary to uraemia).
\end{abstract}

(Ann Rheum Dis 1997;56:393-394)

Systemic sclerosis is a multi-system disease characterised by dense fibrosis of visceral organs and skin. ${ }^{1}$ It commonly involves the heart, causing myocardial fibrosis and cardiac failure. ${ }^{23}$ Necropsy studies report pericardial involvement in between $33 \%$ and $72 \%$ of cases $^{24-7}$ but symptoms from pericarditis occur in only $7 \%$ to $20 \%$ of patients. ${ }^{67}$ Previous necropsy studies have relied on macroscopic inspection ${ }^{24-7}$ and have not examined the pericardium histologically. We investigated the pericardial changes at the histological level, and, as mast cells have been implicated in the development of fibrosis in systemic sclerosis, ${ }^{8-10}$ also studied the level of mast cell infiltration in the pericardium in systemic sclerosis.

\section{Methods}

Forty four postmortem specimens of cardiac tissue (26 female, 18 male), from patients dying in hospital between 1971 and 1991 were obtained from pathology departments throughout the United Kingdom. All patients had a diagnosis of scleroderma recorded on the death certificate and postmortem reports were examined where available. A control group of 19 age/sex matched patients dying without evidence of connective tissue, heart or renal disease was retrieved from the files of Manchester Royal Infirmary.

Blocks of cardiac tissue containing overlying pericardium were selected from each case in both groups and sections stained with haematoxylin and eosin, acid toluidine blue, for mast cells, and elastic van Gieson, for collagen and elastic fibres. The histological features were reviewed in each case and the presence of pericardial inflammation and fibrosis and of any myocardial changes noted.

Mast cells, disclosed by the acid toluidine blue stain, were counted in 10 high power fields (hpf) at the pericardial surface in each group. The degree of fibrosis in each group was quantified using a 25 point Chalkley point eyepiece graticule. ${ }^{11}$ Points lying over collagen fibres, indicated by the elastic van Gieson stain, were counted in $10 \mathrm{hpf}$, so that the count in each case was out of a maximum number of 250 points.

\section{Results}

The patients ranged in age from 9 to 89 years (mean 54 in males and 41 in females), while the controls (9 males, 10 females) ranged in age from 26 to 84 years (mean 52.5 in males and 49.8 in females). Myocardial fibrosis was seen in $15(37.5 \%)$ of the systemic sclerosis cases but in none of the control group while pericarditis was present in $31(77.7 \%)$ of those with systemic sclerosis, but in only one of the controls. The pericardial infiltrate was composed predominantly of chronic inflammatory cells in most cases (fig 1), though two showed an additional neutrophilic infiltrate. Dense fibrinous deposits, characteristic of uraemic pericarditis, were seen in two cases, one of which had confirmed evidence of renal failure.

The amount of pericardial fibrosis in those with systemic sclerosis was significantly greater than in the controls with a mean Chalkley

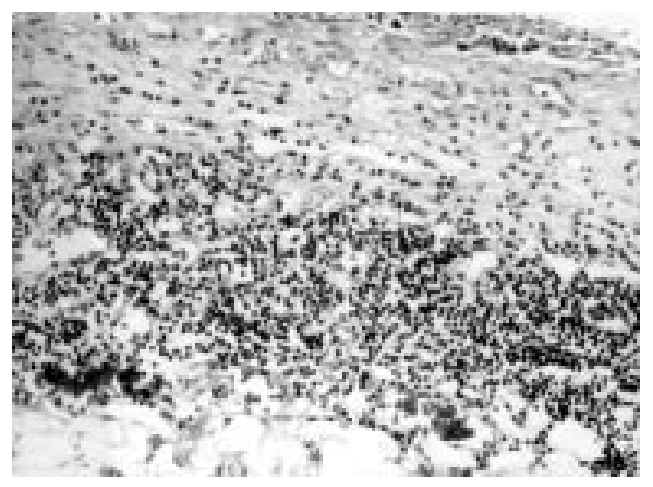

Figure 1 Pericardial fibrosis and infiltrate of chronic inflammatory cells. Haematoxylin and eosin, original magnification $\times 40$. 
count of 21.1 of 250 compared with 11.8 of $250(\mathrm{p}<0.05)$. The incidence of mast cells was also greater in those with systemic sclerosis, with a mean of 4.1 mast cells $/ 10 \mathrm{hpf}$ in the index group and $2.9 / 10 \mathrm{hpf}$ in the controls, but the difference was not significant $(p>0.05)$. Acute myocardial infarction was seen in three cases. Detailed postmortem reports were available in 16 cases, of which eight had confirmed renal failure, with uraemia, at death.

\section{Discussion}

Systemic sclerosis causes visceral fibrosis and frequently results in cardiac failure caused by myocardial fibrosis. ${ }^{1}$ Pericardial involvement has been reported in between $33 \%$ and $72 \%$ of cases in necropsy series, ${ }^{24-7}$ and includes fibrinous pericarditis, chronic fibrous pericarditis, pericardial adhesions, and pericardial effusions. Most cases are asymptomatic, with premorbid symptoms being reported in only $7 \%$ to $20 \%$ of patients, ${ }^{67}$ though echocardiographic studies demonstrate the higher prevalence of subclinical pericardial involvement in systemic sclerosis. ${ }^{12}{ }^{13}$ None of the previous reports has included histological assessment of the pericardium. Furthermore, though in most cases the pericardial changes seen are primary, it has been suggested that in some cases they are secondary to uraemia, which is frequently seen in end stage systemic sclerosis. $^{7}$

This study confirms the high incidence of pericardial disease reported previously. The inflammation was chronic in all the cases, with an additional neutrophilic infiltrate in two cases, and was accompanied by a significant increase in fibrous tissue compared with controls, in keeping with macroscopic reports of an increase in pericardial adhesions. The characteristic fibrinous exudate and acute inflammatory response seen in uraemic pericarditis was seen in only two cases, one of whom had reported evidence of renal failure, and, in the subgroup of patients for whom necropsy reports were available, the degree of fibrosis was similar in those with reported renal failure and in those without (mean Chalkley counts of 15.3 and 14.5 respectively). This suggests that in most cases the changes seen resulted from primary pericardial involvement by systemic sclerosis rather than uraemia.

Mast cells are thought to be important in the development of fibrosis in systemic sclerosis, ${ }^{8-10}$ via release of basic fibroblast growth factor ${ }^{10}$ and histamine, ${ }^{14}$ which may promote fibroblast proliferation, though other cells including lymphocytes also play a part. Increased numbers of mast cells have been reported in toluidine blue stained tissue sections of dermis of early skin lesions in systemic sclerosis, though the number was equal to controls in late cases, suggesting that the primary role is in the early phase of the disease. ${ }^{9}$ Toluidine blue detects only undegranulated mast cells. Recently a study using immunofluorescence to detect degranulated, 'phantom', mast cells demonstrated large numbers of degranulated mast cells in the dermis in systemic sclerosis. ${ }^{15}$ The same study found that mast cell degranulation precedes fibrosis, and indicated that degranulation is not specific for systemic sclerosis, but may occur in other situations of severe illness. ${ }^{14} 15$ Although we found an increase in pericardial mast cells in those with systemic sclerosis compared with controls the increase did not reach statistical significance. The cases in this study were obtained at necropsy and had longstanding disease. Thus changes in mast cell number in early disease may no longer have been present. Alternatively, as the method used detected only granulated mast cells, it is possible that the number has been underestimated, and that larger numbers of degranulated mast cells are present, degranulation being precipitated by severe illness before death or the process of systemic sclerosis.

While our patients are a selected cohort of systemic sclerosis patients we believe this postmortem study has emphasised a comparatively common feature of the pathology of systemic sclerosis. That antemortem echocardiography has also shown a high incidence of pericardial involvement ${ }^{12}$ suggests that this finding is not restricted to those dying with the disorder. The pericardial changes are those of fibrosis and chronic inflammation, while mast cell numbers were not significantly increased compared with normal controls. The pericardial changes are not typical of uraemia and we believe they reflect primary involvement in the disease process rather than those secondary to uraemia. The lack of increase in mast cells may reflect the late stage of the disease, or degranulation caused by severe illness in these cases.

This work was supported by the Arthritis and Rheumatism Council for Research.

1 LeRoy EC. Scleroderma (systemic sclerosis). In: Kelly WN, Harris JR, Ruddy S, Sledge CB, eds. Textbook of rheumatology. Philadelphia: W B Saunders, 1981:1211-30.

2 Buckley BH, Ridolfi RL, Salyer WR, Hutchins GM. Myocardial lesions of progressive systemic sclerosis. Circulation 1976;53:483-90.

3 Botstein GR, LeRoy EC. Primary heart disease in systemic sclerosis (scleroderma): advances in clinical and pathological features, pathogenesis, and new therapeutic approaches. Am Heart J 1981;102:913-9.

4 Oram S, Stokes W. The heart in scleroderma. Br Heart J 1961;23:243-59.

5 Sackner MA, Heinz ER, Steinberg AJ. The heart in scleroderma. Am J Cardiol 1966;17:542-59.

6 D'Angelo WA, Fries JF, Masi AT, Shulman LE. Pathologic observations in systemic sclerosis (scleroderma). Am J Med 1969;46:428-40.

7 McWhorter JE, LeRoy EC. Pericardial disease in scleroderma ( systemic sclerosis). Am J Med 1974;57:566-75.

8 Lichtbroun AS, Sandhaus LM, Giorno RC, Kim H, Siebold JR. Myocardial mast cells in systemic sclerosis: a report of three fatal cases. Am J Med 1990;89:372-6.

9 Hawkins RA, Claman HN, Clark RAF, Steigerwald JC. Increased dermal mast cell populations in progressive systemic sclerosis: a link in chronic fibrosis? Ann Intern Med 1985;102:182-6.

10 Qu Z, Liebler JM, Powers MR, Galey T, Ahmadi P, Huang $\mathrm{X}-\mathrm{N}$, et al. Mast cells are a major source of basic fibroblast growth factor in chronic inflammation and cutaneous hemangioma. Am J Pathol 1995;147:564-73.

11 Chalkley $\mathrm{H}$. Method for the quantitative morphological analysis of tissues. J Natl Cancer Inst 1943;4:47-53.

12 Smith JW, Clements PJ, Levisman J, Furst D, Ross $M$ Echocardiographic features of progressive systemic scleroEchocardiographic features of progressive systemic sclero-
sis (PSS): correlation with hemodynamic and postmis (PSS): correlation with hemodynamic

13 Andrias CW. Scleroderma heart disease. Conn Med 1985;49:214-8.

14 Lee Choi K, Claman HN. Mast cells, fibroblasts, and fibrosis: new clues to the riddle of mast cells. Immunol Res 1987;6:145-52.

15 Seibold JR, Giorno RC, Claman HN. Dermal mast cell degranulation in systemic sclerosis. Arthritis Rheum 1990; 33:1702-9. 